\title{
Application Research of the Gear's Predictor- Corrector Algorithms in A Molecular Dynamics Simulation to the EXP-6 Potential Function of Liquid Helium
}

\author{
Chen $\mathrm{Yu}$ \\ College of Mechanical Engineering \\ Shanghai University of Engineering Science \\ Shanghai, China \\ e-mail: yvettee1976@126.com
}

\author{
Chen Shuo \\ School of Aerospace Engineering and Applied \\ Mechanics \\ Tongji University \\ Shanghai, China \\ schen_tju@tongji.edu.cn
}

\begin{abstract}
Molecular dynamics (MD) is a method for computationally evaluating the thermodynamic and transport properties of materials by solving the classical equations of motion at the molecular level. There are many algorithms that can be used to integrate the Newton's equation of motion in the simulation process. Each has its own advantages and disadvantages. GPC method keeps track of several time derivatives of the particle positions. Moreover, the GPC method requires only one evaluation of the forces per time step. This is of chief importance because we will find that the evaluation of forces is the most computationally expensive part of the molecular dynamics simulation. Therefore, the intermolecular interaction resulted from the potentials is very important for the molecular reaction dynamics and Molecular Dynamics. For helium, the repulsive part of the potential expressed in minus twelve power of $r$ is not fit for it and exponential function form is used instead. EXP-6 potential is one of the potential functions with exponential repulsive interaction could explain the multi-body interaction between the liquid helium atoms and the attractive part is the main part of the Van der Waals attractive interaction. The behavior of GPC algorithm is observed during a canonical MD simulation of liquid helium. The relationship between the stability and accuracy of the GPC algorithm and the size of the time step will be explained in detail.
\end{abstract}

Keywords- Gear's predictor-corrector algorithms; Molecular dynamics; potential function; liquid helium; simulation

\section{INTRODUCTION}

Molecular dynamics (MD) has about 45 years of research history since its development from 1966, with the rapid development of computer technology and MD algorithm research, MD simulation studies has been applied to various fields and systems. The core of this simulation technique is by solving the equation of motion of all the particles in the initial structure, the basic procedure of physical information related to the particle motion path can be obtained. In fact, in a molecular dynamics simulation, many integration algorithm for solving Newton's equation of motion can be chose, we need to select the appropriate algorithm based on the calculation purposes.

In a complete MD simulation process, the force calculation steps, calculating the force acting on each particle, is the most time-consuming part of almost all of the molecular dynamics simulation process. Therefore, in the integration algorithms, each integral step which need to calculate the potential energy twice to get the interaction forces between the atoms, but cannot guarantee an appropriate proportional increased time step under the same calculation accuracy is undesirable ${ }^{[1]}$. Therefore, there are two types of technologies have been widely used, one is the low-level leapfrog algorithm, the algorithm has a simple mathematical form and has good energy conservation features; the other is the predictor-corrector algorithm. Under normal conditions, the predictorcorrector algorithm requires the calculation of the potential energy of the simulation system twice in order to obtain the interaction force between atoms within each integration step, but which allows the time step is more than twice longer than the other algorithms.

\section{GEAR PREDICTOR-CORRECTOR ALGORITHM}

Gear predictor-corrector (GPC) is a method that allows us to numerically solve a second-order ODE without converting it into a system of first order ODEs. Moreover, the GPC method requires only one evaluation of the forces per time step. This is of chief importance because we will find that the evaluation of forces is the most computationally expensive part of the molecular dynamics simulation.

The basic idea of GPC algorithm is Taylor series expansion and the algorithm is one of the commonly used integration algorithms in the MD simulation process. Here we describe a fifth-order GPC method appropriate for solving a second-order ordinary differential equations 
(ODEs). The ODEs is second order because it has a second derivative in time. The order is fifth order because it is based on a Taylor series that includes all terms out to the fifth derivative. The GPC method usually consists of three parts: the first part, which uses the Taylor series expansion to predict the locations $r$, and first to fifth derivatives of the particles at the next time, are shown in equations (1),

$$
\begin{gathered}
r^{p}(t+\delta t)=r(t)+v(t) \delta t+\frac{1}{2} \delta t^{2} a(t)+\frac{1}{6} \delta t^{3} b(t)+\frac{1}{24} \delta t^{4} c(t)+\frac{1}{120} \delta t^{5} d(t)+\Lambda \\
v^{p}(t+\delta t)=v(t)+\delta t a(t)+\frac{1}{2} \delta t^{2} b(t)+\frac{1}{6} \delta t^{3} c(t)+\frac{1}{24} \delta t^{4} d(t)+\Lambda \\
a^{p}(t+\delta t)=a(t)+\delta t b(t)+\frac{1}{2} \delta t^{2} c(t)+\frac{1}{6} \delta t^{3} d(t)+\Lambda \\
b^{p}(t+\delta t)=b(t)+\delta t c(t)+\frac{1}{2} \delta t^{2} d(t)+\Lambda \\
c^{p}(t+\delta t)=c(t)+\delta t d(t)+\Lambda
\end{gathered}
$$

where $v, a, b 、 c 、 d$ are the positions and the derivatives of all the particles at next time, the superscripts $\mathrm{p}$ represents the predictive value (predictor). The second step of the GPC is based on a new atomic position, i.e. the predicted values obtained in the first step to calculate the forces and the corrected acceleration term $a^{c}(t+\delta t)$. Then defining the prediction error as equation (2) .

$$
\Delta a(t+\delta t)=a^{c}(t+\delta t)-a^{p}(t+\delta t)
$$

The third part is the correction step. The corrected acceleration $a^{c}(t+\delta t)$ is used to correct the position and its derivatives based on the predicted positions. The last step is shown in equation (3).

$$
\begin{aligned}
& r^{c}(t+\delta t)=r^{p}(t+\delta t)+c_{0} \Delta a(t+\delta t) \\
& v^{c}(t+\delta t)=v^{p}(t+\delta t)+c_{1} \Delta a(t+\delta t) \\
& a^{c}(t+\delta t)=a^{p}(t+\delta t)+c_{2} \Delta a(t+\delta t) \\
& b^{c}(t+\delta t)=b^{p}(t+\delta t)+c_{3} \Delta a(t+\delta t) \\
& c^{c}(t+\delta t)=c^{p}(t+\delta t)+c_{4} \Delta a(t+\delta t) \\
& d^{c}(t+\delta t)=d^{p}(t+\delta t)+c_{5} \Delta a(t+\delta t)
\end{aligned}
$$

The values of the corrector coefficients of $c_{0} 、 c_{1}$ 、 $c_{2} 、 c_{3} 、 c_{4} 、 c_{5}$ depend upon the order of the ODEs, the order of the GPC method, and the functional form of the ODEs. They are selected to maximize stability and minimize error. For a second-order ODEs with a fifthorder GPC method, where the forces are strictly a function of position the corrector coefficients are listed in Table 1.
TABLE I. CORRECTOR COEFFICIENTS

\begin{tabular}{|c|c|c|c|c|c|}
\hline$c_{0}$ & $c_{1}$ & $c_{2}$ & $c_{3}$ & $c_{4}$ & $c_{5}$ \\
\hline $3 / 20$ & $251 / 360$ & 1 & $11 / 18$ & $1 / 6$ & $1 / 60$ \\
\hline
\end{tabular}

\section{THE EXP-6 POTENTIAL FUNCTION FOR HELIUM}

General form of the exponential potential function, which can be used to describe the interaction between the helium atoms, may be constructed by the Van der Waals attractive interaction potential and exponential repulsive interaction. The general form of the exponential potential function can be written as ${ }^{[2-4]}$,

$$
u(r)=A e^{-B \times r}-\frac{C}{r^{6}}-\frac{D}{r^{8}}-\frac{E}{r^{10}}-\Lambda
$$

The first item of the equation (4) is the repulsive interaction, the second, third and fourth items are attractive potential caused by the dipole interaction, dipole - quadrupole interaction and quadrupole - quadrupole interaction, respectively. In the practical application of the molecular dynamics the multi-polar interaction is often ignored, so the EXP-6 potential function with forms such as formula (5) is obtained.

$$
u(r)=\varepsilon\left[\frac{6}{\alpha-6} e^{\alpha\left(1-r / r_{m}\right)}-\frac{\alpha}{\alpha-6}\left(\frac{r_{m}}{r}\right)^{6}\right]
$$

$u(r)$, which is the function of the distance between the helium atoms represents the helium potential energy, $\varepsilon$ is the potential well, and $r_{m}$ is the distance between the helium atoms when the potential energy is $\varepsilon$. The parameter $\alpha$ in the equation (5) is the repulsive potential slope of the potential function. Formula (5) is the typical form of the EXP-6 potential function for helium, which is composed of two parts. The exponential repulsive interaction part is obtained by the quantum mechanical calculations. The other part is Van der Waals attractive 
potential expressed by $\mathrm{r}^{-6}$, which is the main representative of the dipole - dipole interaction. The interactions between the dipole - quadrupole and quadrupole - quadrupole and other multi-polar interactions are ignored ${ }^{[5-13]}$.

Huijper ${ }^{[14]}$ proved that the EXP-6 potential function with three parameters could reflect the many-body interactions between the liquid helium atom, and it is one of the most accurate expressions of the form of the potential energy function describing helium characteristics. Typically, material compression factor and other transmission parameters can be used to determine the parameters in formula (5). Mason[2] had used a relatively simple second Virial coefficients to calculate the parameters for helium, the results are shown in Table 2.

TABLE II. POTENTIAL PARAMETERS FOR THE EXP-6 AND STANDARD LJ POTENTIAL FOR HELIUM

\begin{tabular}{|c|c|}
\hline Parameters & Values in EXP-6 potential function \\
\hline$\alpha$ & 12.4 \\
\hline$r_{m} / \AA$ & 3.135 \\
\hline$\varepsilon / k_{B}(\mathrm{~K})$ & 9.16 \\
\hline
\end{tabular}

\section{THE RELATIONSHIP BETWEEN THE STABILITY OF THE GPC ALGORITHM AND THE SIZE OF THE TIME STEP}

In a standard (Micro-canonical: specify $\mathrm{N}, \mathrm{V}, \& \mathrm{E}$ ) molecular dynamics simulation, we do not specify the pressure. Therefore, the best we can do here is specifying a density. To do this we can, for example, use the particle density and Avogadro constant to predict the liquid density (units of molecules per $\AA^{3}$ ) for helium at T $=2.2 \mathrm{~K}, 3$ and $3.5 \mathrm{~K}$. The densities are 0.022, 0.0213, and 0.0205 molecules $/ \AA^{3}$ for system of $\mathrm{N}=108$ molecules yields a system volume are 4914, 5076 and $5278 \AA^{3}$, respectively. We equilibrate this system for 2500 equilibrium steps and produce data for 2000 data production. In Table 3, we compare the thermodynamic properties from NIST Technical Note $1334^{[15]}$ and the simulation.

TABLE III. COMPARISON OF THEORETICAL AND SIMULATION THERMODYNAMIC PROPERTIES FOR LIQUID AT DIFFERENT TEMPERATURE AND DENSITIES

\begin{tabular}{|c|c|c|c|c|c|}
\hline $\begin{array}{c}\mathrm{T} \\
(\mathrm{K})\end{array}$ & $\begin{array}{c}\text { System } \\
\text { volume } \\
\left(\AA^{3}\right)\end{array}$ & $\begin{array}{c}\text { Particles } \\
\text { density } \\
\text { (molecules / } \\
\AA^{3} \text { ) }\end{array}$ & $\begin{array}{l}\text { Total } \\
\text { energy- } \\
\text { theory } \\
(\mathrm{aJ})\end{array}$ & $\begin{array}{c}\text { Total } \\
\text { energy- } \\
\text { simulation } \\
(\mathrm{aJ})\end{array}$ & $\begin{array}{l}\text { Time } \\
\text { step } \\
\text { (fs) }\end{array}$ \\
\hline 2.2 & 4914 & 0.022 & $\begin{array}{c}-5.3 \times 10^{-} \\
-\end{array}$ & $5.316 \times 10^{-3}$ & 1 \\
\hline 3 & 5076 & 0.0213 & $\begin{array}{c}-3.6 \times 10^{-} \\
3\end{array}$ & $-3.6088 \times 10$ & 0.5 \\
\hline 3.5 & 5278 & 0.0205 & $-2.6 \times 10^{-}$ & $-2.523 \times 10^{-3}$ & 0.08 \\
\hline
\end{tabular}

Note: $\AA$, angstrom $\left(=10^{-10} \mathrm{~m}\right) ; \mathrm{aJ},\left(=10^{-18} \mathrm{~J}\right) ; \mathrm{fs},\left(=10^{-15} \mathrm{~s}\right)$
From the Table 3, we can see that the accuracy and stability of the simulation results are related to the time step. With the simulated temperature goes down, the time step required is larger.

\section{CONCLUSION}

GPC method is a high-order integrator. It is importance that the GPC method requires only one evaluation of the forces per time step. For helium, the repulsive part of the potential expressed in minus twelve power of $r$ is not fit for it and exponential function form is used instead. EXP-6 potential is one of the potential functions with exponential repulsive interaction could explain the multi-body interaction between the liquid helium atoms and the attractive part is the main part of the Van der Waals attractive interaction. We carried out a standard (Microcanonical: specify $\mathrm{N}, \mathrm{V}, \& \mathrm{E})$ molecular dynamics simulation and compared the thermodynamic properties from NIST Technical Note 1334 and simulated results. From comparing the results we can see that the accuracy and stability of the simulation results are related to the time step. With the simulated temperature goes down, the required time step is larger.

\section{ACKNOWLEDGMENT}

This work is supported by the Chinese National Natural Science Foundation (Foundation No. 51276130).and Shanghai Natural Science Foundation (Foundation No.10ZR1412700)

\section{REFERENCES}

[1] D.C.Rapaport, The art of molecular dynamics simulation, Second Edition, Cambridge University Press, 2004

[2] Mason E.A., Rice W.E., The intermolecular potentials of helium and hydrogen. The Journal of Chemical Physics, 1954, 22(3): 522535.

[3] Rice W.E., Hirschfelder J.O., Second virial coefficients of gases obeying a modified Buckingham (Exp-Six) potentials. The Journal of Chemical Physics, 1954, 22(2): 187-192.

[4] Mason E.A., Transport properties of gases obeying a modified Buckingham (Exp-Six) potential. The Journal of Chemical Physics, 1954, 22(2): 169-186.

[5] Chester H. P., Van der Waals forces in helium. Physical Review, 1938, 53(1): 426-430

[6] Henry Margenau, The role of quadrupole forces in Van der Waals attractions. Physical Review, 1931, 38(15): 747-756

[7] Slater J.C., Kiekwood J.C., The Van ver Waals forces in gases. Physical Review, 1931, 37(15): 682-695

[8] Slater J.C., The normal state of helium. Physical Review, 1928 , 32(9): 349-360

[9] Margenau H., Van der Waals potential in helium. Physical Review, 1939, 56(15): 1000-1008

[10] Amdur I., Pearlman H., Helium repulsive potential from collision cross section measurements. The Journal of Chemical Physics, 1941, 9(6): 503-506

[11] Amdur I., Repulsive interaction potentials at small interaction distances: $\mathrm{He}-\mathrm{He}$ and $\mathrm{H}-\mathrm{H}_{2}$ systems. The Journal of Chemical Physics, 1949, 17: 844-845

[12] Amdur I., Harkness A.L., Scattering of high-velocity neutral particles. II. Helium-Helium. The Journal of Chemical Physics, 1954, 22(4): 664-669

[13] Rosen P., The repulsive potential of normal helium atoms. The Journal of Chemical Physics, 1950, 18(9): 1182-1186 
[14] De Kuijper A., Vos W. L., Barrat J L, et al., Freezing of simple system using density function theory. The Journal of chemical physics, 1990, 93(7): 5187-5193
[15] Vincent D. Arp, Robert D. McCarty, Daniel G. Friend, National Institute of Standards and Technology Technical Note, U.S. Government printing Office, Washington, 1998 